\title{
LIGHTLIKE HYPERSURFACES OF METALLIC SEMI-RIEMANNIAN MANIFOLDS
}

\author{
BİLAL EFTAL ACET
}

\begin{abstract}
In our article, we introduce and study lightlike hypersurfaces of a metallic semi-Riemannian manifold. We examine some geometric properties of invariant lightlike hypersurfaces. We show that the induced structure on an invariant lightlike hypersurface is also metallic. We also define screen semiinvariant lightlike hypersurfaces, investigate integrability conditions for the distributions and give some examples.
\end{abstract}

\section{INTRODUCTION}

In terms of differential geometry, submanifold theory has an attraction for geometers. One of the most important topic is the theory of lightlike submanifolds. A submanifold of a semi-Riemann manifold is called a lightlike submanifold if the induced metric is degenerate. So, geometry of lightlike submanifold is very different from the non-degenerate submanifold. The general view of lightlike submanifold has been introduced in [1. Later, K. L. Duggal and B. Şahin have been developed many new classes of lightlike submanifolds on indefinite Kaehler [2], indefinite Sasakian [3] manifolds and different applications of lightlike submanifolds [4. On this subject, some applications of the theory of mathematical physics, especially electromagnetisms [1, black hole theory [4] and general relativity [5], is inspired. Many studies on lightlike submanifolds have been reported by many geometers (see [8, 9, 14, 12, 19, 11, 10, and the references therein).

Investigating submanifold theory on manifolds endowed with various geometric structures provides a fruitful study field. Recently, Riemannian manifolds with metallic structures are widely studied and metallic structures on Riemannian manifolds provides many geometric results to characterize a submanifold of such ambient manifolds.

As a generalization of the golden mean, in 2002, V. W. de Spinadel 16] introduced metallic means family which contains the silver mean, the bronze mean, the copper mean and the nickel mean etc. For some positive integer $p$ and $q$, the positive solution of

$$
x^{2}-p x-q=0,
$$

is called a $(p, q)$-metallic number [15] which has the expression

$$
\sigma_{p, q}=\frac{p+\sqrt{p^{2}+4 q}}{2}
$$

2010 Mathematics Subject Classification. 53C15, 53C25, 53C 35.

Key words and phrases. Metallic structure, Lightlike hypersurface, Screen semi-invariant lightlike hypersurface. 
It is well-known that we have the golden mean $\phi=\frac{1+\sqrt{5}}{2}$, for $p=q=1$ and the silver mean $\sigma_{2,1}=1+\sqrt{2}$, for $p=2, q=1$. The metallic mean family plays an important role to establish a relationship between mathematics and architecture. For example silver and golden mean can be seen in the sacred art of India, Egypt, China, Turkey and different ancient civilizations [20.

Polynomial structures on manifolds were introduced by S. I. Goldberg, K. Yano and N. C. Petridis in 21, 22. As a particular case of polynomial structures which is called golden structure was defined in [24, 25] and some generalizations of this called metallic structure in [26. Being inspired by the metallic mean, the term of metallic manifold was studied in [26] by a $(1,1)$-tensor field $\tilde{J}$ on $\tilde{N}$ which satisfies $\tilde{J}^{2}=p \tilde{J}+$ $q I$, where $p, q$ are fixed positive integer numbers and $I$ is the identity operator on $\tilde{N}$. Moreover, if $(\tilde{N}, \tilde{g})$ is a Riemannian manifold endowed with a metallic structure

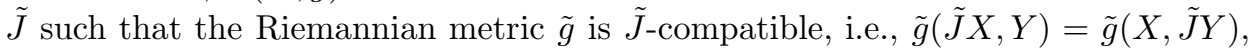
for any $X, Y \in X(\tilde{N})$, then $(\tilde{g}, \tilde{J})$ is called metallic Riemannian structure and $(\tilde{N}, \tilde{g}, \tilde{J})$ is a metallic Riemannian manifold. Metallic structure on the ambient Riemannian manifold provides important geometrical results on the submanifolds, since it is an important tool while investigating the geometry of submanifolds. Invariant, anti-invariant, semi-invariant, slant and semi-slant submanifolds of a metallic Riemannian manifold are studied in 28, 29, 30 and the authors obtained important characterizations on submanifolds of metallic Riemannian manifolds.

One of the most important subclasses of metallic Riemannian manifolds is the golden Riemannian manifolds. Many authors have studied golden Riemannian manifolds and their submanifolds in recent years (see [25, 27, 18, 7]. N. Poyraz Önen and E. Yaşar [13] initiated the study of lightlike geometry in Golden semi-Riemannian manifolds, by investigating lightlike hypersurfaces of a golden semi-Riemannian manifold.

Motivated by the studies on submanifolds of metallic Riemannian manifolds and lightlike submanifolds of semi-Riemannian manifolds, in this paper we introduce lightlike hypersurfaces of a metallic semi-Riemannian manifold. Section 1 is devoted to preliminaries containing basic definitions for metallic semi-Riemannian manifolds and lightlike hypersurfaces. Section 3 is divided two subsections. Firstly we define invariant lightlike hypersurfaces of a metallic semi-Riemannian manifold and prove that induced structures on the invariant hypersurfaces are also metallic. In Subsection 2 we examine screen semi-invariant lightlike hypersurfaces, give examples and investigate integrability conditions for the distributions defined in such hypersurfaces.

\section{Preliminaries}

The positive solution of

$$
x^{2}-p x-q=0,
$$

is called member of the metallic means family [16], where $p, q$ are fixed positive integers. These numbers denoted by;

$$
\sigma_{p, q}=\frac{p+\sqrt{p^{2}+4 q}}{2}
$$

are known $(p, q)$-metallic numbers. 
A polynomial structure on a semi-Riemannian manifold $\tilde{N}$ is known metallic if it is determined by $\tilde{J}$ such that

$$
\tilde{J}^{2}=p \tilde{J}+q I
$$

If a semi-Riemannian metric $\tilde{g}$ satisfies the equation

$$
\tilde{g}(U, \tilde{J} V)=\tilde{g}(\tilde{J} U, V)
$$

which yields

$$
\tilde{g}(\tilde{J} U, \tilde{J} V)=p \tilde{g}(U, \tilde{J} V)+q \tilde{g}(U, V),
$$

then $\tilde{g}$ is called $\tilde{J}$-compatible.

Definition 2.1. A semi-Riemannian manifold $(\tilde{N}, \tilde{g})$ equipped with $\tilde{J}$ such that the semi-Riemannian metric $\tilde{g}$ is $\tilde{J}$-compatible is named a metallic semi-Riemannian manifold and $(\tilde{g}, \tilde{J})$ is called a metallic semi-Riemannian structure on $\tilde{N}$.

Example 2.1. Let $\tilde{N}=\mathbb{R}_{3}^{7}$ be a semi-Euclidean space with coordinate system $\left(x_{1}, x_{2}, \ldots, x_{7}\right)$ and signature $(-,+,-,+,-,+,+)$.

Taking

$$
\tilde{J}\left(x_{1}, x_{2}, \ldots, x_{7}\right)=\left((p-\sigma) x_{1}, \sigma x_{2},(p-\sigma) x_{3}, \sigma x_{4},(p-\sigma) x_{5}, \sigma x_{6}, \sigma x_{7}\right),
$$

then we can see that

$$
\tilde{J}^{2}=p \tilde{J}+q I .
$$

Therefore $\tilde{J}$ is a metallic structure on $\tilde{N}$.

Remark 2.1. Putting $p=1=q$ in (2.5) then $(\tilde{g}, \tilde{J})$ is a golden (semi)-Riemannian structure (see [6], [7]).

A polynomial structure on $\tilde{N}$ defined by a smooth tensor field of type $(1,1)$ induces a generalized almost product structure $\tilde{F}, \tilde{F}^{2}=I$, on $\tilde{N}$.

Proposition 2.1. 26] Every almost product structure $\tilde{F}$ induces two metallic structure on $\tilde{N}$ given as follow:

$$
\tilde{J}_{1}=\frac{p}{2} I+\left(\frac{2 \sigma_{p, q}-p}{2}\right) \tilde{F}, \quad \tilde{J}_{2}=\frac{p}{2} I-\left(\frac{2 \sigma_{p, q}-p}{2}\right) \tilde{F} .
$$

Conversely, every metallic structure $\tilde{J}$ on $\tilde{N}$ induces two almost product structures:

$$
\tilde{F}= \pm\left(\frac{2}{2 \sigma_{p, q}-p} \tilde{J}-\frac{p}{2 \sigma_{p, q}-p} I\right) .
$$

Let $\tilde{N}$ be a semi-Riemannian manifold with index $q, 0<q<n+1$, and $N$ be a hypersurface of $\tilde{N}$, with $g=\left.\tilde{g}\right|_{\hat{N}}$. Then $N$ is a lightlike hypersurface of $\tilde{N}$, if the metric $g$ is of rank $n$ and the orthogonal complement $T N^{\perp}$ of $T \hat{N}$, given as

$$
T \hat{N}^{\perp}=\bigcup_{p \in \hat{N}}\left\{V_{p} \in T_{p} \tilde{N}: g_{p}\left(U_{p}, V_{p}\right)=0, \forall U \in \Gamma\left(T_{p} N^{\prime}\right)\right\},
$$

is a distribution of rank 1 on $N^{1}$ [1. $T N^{\perp} \subset T N$ and then it coincides with the radical distribution $\operatorname{RadTN}=T N^{\prime} \cap T N^{\perp}$.

A complementary bundle of $T N^{\perp}$ in $T N$ is a non-degenerate distribution of constant rank $n-1$ over $N$, which is known a screen distribution and demonstrated with $S(T N)$. 
Theorem 2.1. 1] Let $(N$, $g, S(T N))$ be a lightlike hypersurface of a semi-Riemannian manifold $\tilde{N}$. Then there exists a unique rank 1 vector sub-bundle $\operatorname{ltr}(T \hat{N})$ of $T \tilde{N}$, with base space $N$, such that for every non-zero section $E$ of RadTŃ on a coordinate neighbourhood $\wp \subset N$, there exists a section $N$ of $l \operatorname{tr}(T N)$ on $\wp$ satisfying:

$$
\tilde{g}(N, N)=0, \quad \tilde{g}(N, W)=0, \quad \tilde{g}(N, E)=1, \quad \text { for } W \in \Gamma\left(\left.S(T N)\right|_{\wp} .\right.
$$

$\operatorname{ltr}\left(T N^{\prime}\right)$ is called the lightlike transversal vector bundle of $N^{\prime}$.

By the previous theorem, we can state:

$$
\begin{aligned}
& T N=S\left(T N^{\prime}\right) \perp \operatorname{RadTN}, \\
T \tilde{N}= & T N{ }^{\prime} \oplus \operatorname{ltr}\left(T N^{\prime}\right) \\
= & S\left(T N^{\prime}\right) \perp\left\{\operatorname{RadTN} \oplus \operatorname{lt}\left(T N^{\prime}\right)\right\} .
\end{aligned}
$$

Let $\omega: \Gamma\left(T N^{\prime}\right) \rightarrow \Gamma\left(S\left(T N^{\prime}\right)\right)$ be the projection morphism. For $U, V \in \Gamma\left(T N^{\prime}\right)$, we have

$$
\begin{gathered}
\tilde{\nabla}_{U} V=\nabla_{U} V+B(U, V) N, \\
\tilde{\nabla}_{V} N=-A_{N} U+\tau(U) N, \\
\nabla_{U} \omega V=\nabla_{U}^{*} \omega V+C(U, \omega V) E, \\
\nabla_{U} E=-A_{E}^{*} U-\tau(V) E .
\end{gathered}
$$

For the induced connection $\nabla$, we have

$$
\left(\nabla_{U} g\right)(V, Z)=B(U, Z) \theta(V)+B(U, V) \theta(Z),
$$

where $\theta$ is a differential 1 -form and

$$
\theta(U)=\tilde{g}(N, U)
$$

Also note that

$$
\begin{gathered}
B(U, E)=0, \\
g\left(A_{E}^{*} U, P V\right)=B(U, P V), \quad g\left(A_{E}^{*} U, N\right)=0, \\
g\left(A_{N} U, P V\right)=C(U, P V), \quad g\left(A_{N} U, N\right)=0, \\
A_{E}^{*} E=0 .
\end{gathered}
$$

\section{LIGHTLIKE HYPERSURFACES OF METALLIC SEMI-RIEMANNIAN MANIFOLDS}

Let $N$ be a lightlike hypersurface of a metallic semi-Riemannian manifold $(\tilde{N}, \tilde{g}, \tilde{J})$. For every $U \in \Gamma(T N)$ and $N \in \Gamma(\operatorname{lt}(T N))$, we get

$$
\begin{gathered}
\tilde{J} U=\varphi U+u(U) N, \\
\tilde{J} N=\xi+v(E) N,
\end{gathered}
$$

where $\varphi U, \xi \in \Gamma\left(T N^{\prime}\right)$, and $u, v$ are 1 -forms given by

$$
u(U)=g(U, \tilde{J} E), \quad v(U)=g(U, \tilde{J} N) .
$$


Lemma 3.1. Let $N$ be a lightlike hypersurface of $(\tilde{N}, \tilde{g}, \tilde{J})$. Then we have

$$
\begin{gathered}
\varphi^{2} U=p \varphi U+q(U)-u(U) \xi, \\
u(\varphi U)=p u(U)-u(U) v(E), \\
\varphi \xi=p \xi-v(E) \xi, \\
v(E)^{2}=p v(E)+q-u(\xi), \\
g(\varphi U, V)=g(U, \varphi V)+u(V) \theta(U)-u(U) \theta(V), \\
g(\varphi U, \varphi V)=\quad p g(U, \varphi V)+q g(U, V)+p u(V) \theta(U) \\
\quad-u(V) g(\varphi U, N)-u(U) g(\varphi V, N) .
\end{gathered}
$$

Definition 3.1. A metallic semi-Riemannain structure $\tilde{J}$ is called a locally metallic structure if $\tilde{J}$ is parallel, i.e., $\tilde{\nabla} \tilde{J}=0$.

Lemma 3.2. Let $N$ be a lightlike hypersurface of a locally metallic semi-Riemannian manifold $(\tilde{N}, \tilde{g}, \tilde{J})$. Then we have

$$
\begin{gathered}
\left(\nabla_{U} \varphi\right) V=u(V) A_{N} U+B(U, V) \xi, \\
\left(\nabla_{U} u\right) V=B(U, V) v(E)-B(U, \varphi V)-\tau(U) u(V), \\
\nabla_{U} \xi=-\varphi A_{N} U+\tau(U) \xi+A_{N} U v(E), \\
U(v(E))=-B(U, \xi)-u\left(A_{N} U\right) .
\end{gathered}
$$

\subsection{Invariant Lightlike Hypersurfaces.}

Definition 3.2. Let $N$ be a lightlike hypersurface of a locally metallic semiRiemannian manifold $(\tilde{N}, \tilde{g}, \tilde{J})$. In that case $N$ is called invariant hypersurface of $\tilde{N}$ if

$$
\begin{aligned}
& \tilde{J}(\operatorname{RadTN})=\operatorname{RadTN}, \\
& \tilde{J}(\operatorname{ltr}(T \hat{N}))=l \operatorname{tr}\left(T N^{\prime}\right) .
\end{aligned}
$$

Example 3.1. Let $\tilde{N}=\mathbb{R}_{2}^{5}$ be a semi-Euclidean space with coordinate system $\left(x_{1}, x_{2}, x_{3}, x_{4}, x_{5}\right)$ and signature $(-,+,-,+,+)$. Taking

$$
\tilde{J}\left(x_{1}, x_{2}, x_{3}, x_{4}, x_{5}\right)=\left(\sigma x_{1}, \sigma x_{2}, \sigma x_{3}, \sigma x_{4}, \sigma x_{5}\right),
$$

then we can see that

$$
\tilde{J}^{2}=p \tilde{J}+q I,
$$

which implies $\tilde{J}$ is a metallic structure on $\tilde{N}$.

Now, consider a hypersurface $N$ of $\tilde{N}$ with

$$
x_{1}=\sigma x_{5} .
$$

Then $T N$ of $N$ is spanned by

$$
\begin{gathered}
\Phi_{1}=\frac{\partial}{\partial x_{2}}, \quad \Phi_{2}=\frac{\partial}{\partial x_{3}} \\
\Phi_{3}=\frac{\partial}{\partial x_{4}}, \quad \Phi_{4}=\sigma \frac{\partial}{\partial x_{1}}+\frac{\partial}{\partial x_{5}}
\end{gathered}
$$


So, $\operatorname{RadTN}$ and $\operatorname{ltr}(T N)$ are given by

$$
\operatorname{RadTN}=\operatorname{Sp}\left\{E=\sigma \frac{\partial}{\partial x_{2}}+\sigma \frac{\partial}{\partial x_{3}}\right\}
$$

and

$$
\operatorname{ltr}\left(T N^{\prime}\right)=S p\left\{N=\frac{1}{2 \sigma^{2}}\left(\sigma \frac{\partial}{\partial x_{2}}-\sigma \frac{\partial}{\partial x_{3}}\right)\right\} .
$$

Thus we arrive at

$$
\tilde{J} E=\sigma E \quad \text { and } \quad \tilde{J} N=\sigma N,
$$

which shows that $N$ is an invariant lightlike hypersurface.

Theorem 3.1. Let $N$ be a lightlike hypersurface of a locally metallic semi-Riemannian manifold $(\tilde{N}, \tilde{g}, \tilde{J})$. Then the structure $\varphi$ is a metallic structure on $N$.

Proof. $N$ is an invariant lightlike hypersurface iff

$$
\tilde{J} U=\varphi U
$$

So we get

$$
u(U)=0 .
$$

By use of (3.4) and (3.8), we arrive at

$$
\varphi^{2} U=p \varphi U+q U
$$

and

$$
g(\varphi U, V)=g(U, \varphi V)
$$

Theorem 3.2. Let $N$ be an invariant lightlike hypersurface of a locally metallic semi-Riemannian manifold $(\tilde{N}, \tilde{g}, \tilde{J})$. Then

$$
\text { i) } B(U, \tilde{J} V)=B(\tilde{J} U, V)=\tilde{J} B(U, V) \text {, }
$$

$$
\text { ii) } B(\tilde{J} U, \tilde{J} V)=p B(U, \tilde{J} V)+q B(U, V) \text {. }
$$

Proof. $i$ ) Since $N$ is an invariant lightlike hypersurface of a locally metallic semiRiemannian manifold $\tilde{N}$, then

$$
\tilde{\nabla}_{U} \tilde{J} V=\nabla_{U} \tilde{J} V+B(U, \tilde{J} V) N
$$

and

$$
\tilde{\nabla}_{U} \tilde{J} V=\tilde{J}\left(\nabla_{U} V\right)+B(U, V) \tilde{J} N .
$$

From (3.18) and (3.19), we obtain (i).

ii) It is obvious from (3.16) with equation (2.5). 


\subsection{Screen Semi-Invariant Hypersurfaces.}

Definition 3.3. Let $N$ be a lightlike hypersurface of a locally metallic semiRiemannian manifold $(\tilde{N}, \tilde{g}, \tilde{J})$. If

$$
\begin{aligned}
& \tilde{J}(\operatorname{RadTN}) \subset S\left(T N^{\prime}\right), \\
& \tilde{J}\left(\operatorname{ltr}\left(T N^{\prime}\right)\right) \subset S\left(T N^{\prime}\right),
\end{aligned}
$$

then $N$ is called a screen semi-invariant hypersurface of $\tilde{N}$.

Example 3.2. Let $\tilde{N}=\mathbb{R}_{2}^{5}$ be a five dimensional metallic semi-Riemannian manifold with the structure $(\tilde{N}, \tilde{g}, \tilde{J})$ given in Example 2.1. Consider a hypersurface $\hat{N}$ of $\tilde{N}$ with

$$
x_{5}=\sigma x_{1}+\sigma x_{2}+x_{3} .
$$

Then $T N^{\prime}$ of $N$ is spanned by

$$
\begin{gathered}
\Phi_{1}=\frac{\partial}{\partial x_{1}}+\sigma \frac{\partial}{\partial x_{5}}, \quad \Phi_{2}=\frac{\partial}{\partial x_{2}}+\sigma \frac{\partial}{\partial x_{5}} \\
\Phi_{3}=\frac{\partial}{\partial x_{3}}+\frac{\partial}{\partial x_{5}}, \quad \Phi_{4}=\frac{\partial}{\partial x_{4}} .
\end{gathered}
$$

The radical distribution $\operatorname{RadTN}$ and lightlike transversal distribution $l \operatorname{tr}(T N)$ are given by

$$
\begin{gathered}
\operatorname{RadTN}=\operatorname{Sp}\left\{E=\sigma \Phi_{1}-\sigma \Phi_{2}+\Phi_{3}\right\}, \\
\operatorname{ltr}\left(T N^{\prime}\right)=S p\left\{N=\frac{1}{2}\left(-\sigma \frac{\partial}{\partial x_{1}}+\sigma \frac{\partial}{\partial x_{2}}-\frac{\partial}{\partial x_{3}}+\frac{\partial}{\partial x_{5}}\right)\right\} .
\end{gathered}
$$

It follows that $S(T N)$ is spanned by $\left\{\Omega_{1}, \Omega_{2}, \Omega_{3}\right\}$, where

$$
\begin{gathered}
\Omega_{1}=-q \frac{\partial}{\partial x_{1}}+q \frac{\partial}{\partial x_{2}}+\sigma \frac{\partial}{\partial x_{3}}+\sigma \frac{\partial}{\partial x_{5}} \\
\Omega_{2}=\frac{1}{2}\left\{\begin{array}{c}
-\sigma(p-\sigma) \frac{\partial}{\partial x_{1}}+\sigma(p-\sigma) \frac{\partial}{\partial x_{2}} \\
-\sigma \frac{\partial}{\partial x_{3}}+\sigma \frac{\partial}{\partial x_{5}}
\end{array}\right\}, \\
\Omega_{3}=\frac{\partial}{\partial x_{4}} .
\end{gathered}
$$

Thus we arrive at

$$
\Omega_{1}=\tilde{J} E \quad \text { and } \quad \Omega_{2}=\tilde{J} N,
$$

which imply that $N$ is a screen semi-invariant hypersurface of $\tilde{N}$.

Example 3.3. Let $\tilde{N}=\mathbb{R}_{2}^{5}$ be a 5-dimensional metallic semi-Riemannian manifold with the structure $(\tilde{N}, \tilde{g}, \tilde{J})$ given in Example 3.1. If we take a hypersurface $N$ of $\tilde{N}$ given by

$$
x_{5}=\sigma x_{3}+\sigma x_{4}+x_{1},
$$

then $T N$ of $N$ is spanned by

$$
\begin{gathered}
\Phi_{1}=\frac{\partial}{\partial x_{1}}+\frac{\partial}{\partial x_{5}}, \quad \Phi_{2}=\frac{\partial}{\partial x_{2}}, \\
\Phi_{3}=\frac{\partial}{\partial x_{3}}+\sigma \frac{\partial}{\partial x_{5}}, \quad \Phi_{4}=\frac{\partial}{\partial x_{4}}+\sigma \frac{\partial}{\partial x_{5}} .
\end{gathered}
$$

The radical distribution $R a d T N$ and lightlike transversal distribution $\operatorname{ltr}(T N)$ are given by

$$
\operatorname{RadTN}=\operatorname{Sp}\left\{E=\sigma \Phi_{3}-\sigma \Phi_{4}+\Phi_{1}\right\},
$$




$$
\operatorname{lt}\left(T N^{\prime}\right)=S p\left\{N=\frac{1}{2}\left(-\frac{\partial}{\partial x_{1}}-\frac{\partial}{\partial x_{3}}+\frac{\partial}{\partial x_{4}}+\frac{\partial}{\partial x_{5}}\right)\right\},
$$

respectively. It follows that $S(T N)$ is spanned by $\left\{\Omega_{1}, \Omega_{2}, \Omega_{3}\right\}$, where

$$
\begin{gathered}
\Omega_{1}=\frac{1}{2}\left\{\begin{array}{c}
-\sigma \frac{\partial}{\partial x_{1}}-\sigma \frac{\partial}{\partial x_{3}} \\
+\sigma \frac{\partial}{\partial x_{4}}+\sigma \frac{\partial}{\partial x_{5}}
\end{array}\right\}, \\
\Omega_{2}=\sigma \frac{\partial}{\partial x_{1}}+\sigma^{2} \frac{\partial}{\partial x_{2}}-\sigma^{2} \frac{\partial}{\partial x_{4}}+\sigma \frac{\partial}{\partial x_{5}}, \\
\Omega_{3}=\frac{\partial}{\partial x_{2}} .
\end{gathered}
$$

Thus we arrive at

$$
\Omega_{1}=\tilde{J} N \quad \text { and } \quad \Omega_{2}=\tilde{J} E,
$$

which imply that $N$ is a screen semi-invariant hypersurface of $\tilde{N}$.

Since $S(T N)$ is non-degenerate, we can define an $(n-2)$-dimensional distribution $\mu_{0}$ such that

$$
S(T N)=\mu_{0} \perp\left\{\tilde{J}(\operatorname{RadTN}) \oplus \tilde{J}\left(l \operatorname{tr}\left(T N^{\prime}\right)\right)\right\},
$$

from which

$$
\begin{gathered}
T N^{\prime}=\left\{\tilde{J}\left(\operatorname{Rad} T N^{\prime}\right) \oplus \tilde{J}\left(\operatorname{ltr}\left(T N^{\prime}\right)\right)\right\} \perp \mu_{0} \perp \operatorname{Rad}\left(T N^{\prime}\right), \\
T \tilde{N}=\left\{\tilde{J}\left(\operatorname{Rad} T \tilde{N}^{\prime}\right) \oplus \tilde{J}\left(\operatorname{ltr}\left(T N^{\prime}\right)\right)\right\} \perp \mu_{0} \perp\left\{\operatorname{Rad}\left(T N^{\prime}\right) \oplus l \operatorname{tr}\left(T N^{\prime}\right)\right\} .
\end{gathered}
$$

Taking $\stackrel{\circ}{D}=\operatorname{Rad}\left(T N^{\prime}\right) \perp \tilde{J}\left(\operatorname{Rad}\left(T N^{\prime}\right)\right) \perp \mu_{0}$ and $\stackrel{\circ}{D}^{\prime}=\tilde{J}\left(\operatorname{ltr}\left(T N^{\prime}\right)\right)$ on $\hat{N}^{\prime}$, we get

$$
T N=\stackrel{\circ}{D} \oplus \stackrel{\circ}{D}^{\prime} .
$$

Let $\zeta=\tilde{J} N$ and $\psi=\tilde{J} E$ be local lightlike vector fields. For $U \in \Gamma(T N)$, we can write

$$
U=Q U+R U,
$$

where $Q$ and $R$ are projections of $T N$ into $\check{D}^{\circ}$ and $\stackrel{\circ}{ }^{\prime}$, respectively.

Moreover, for $U, V \in \Gamma\left(T N^{\prime}\right), \zeta \in \Gamma\left(\stackrel{\circ}{D}^{\prime}\right)$ and $\psi \in \Gamma(\stackrel{\circ}{D})$, we get

$$
\begin{gathered}
\varphi^{2} U=p \varphi U+q(U)-u(U) \zeta, \\
u(\varphi U)=p u(U), \quad u(\zeta)=q, \\
g(\varphi U, V)=g(U, \varphi V)+u(V) \theta(U)-u(U) \theta(V), \\
g(\varphi U, \varphi V)=p g(U, \varphi V)+q g(U, V)+p u(V) \theta(U) \\
-u(V) g(\varphi U, N)-u(U) g(\varphi V, N), \\
\left(\nabla_{U} \varphi\right) V=u(V) A_{N} U+g\left(A_{E}^{*} U, V\right) \zeta, \\
\left(\nabla_{U} u\right) V=-B(U, \varphi V)-u(V) \tau(U), \\
\nabla_{U} \zeta=-\varphi A_{N} U+\tau(U) \zeta, \\
\nabla_{U} \psi=-\varphi A_{E}^{*} U-\tau(U) \psi, \\
B(U, \zeta)=-C(U, \psi) .
\end{gathered}
$$


Theorem 3.3. Assume that $N$ is a screen semi-invariant lightlike hypersurface of a locally metallic semi-Riemannian manifold $(\tilde{N}, \tilde{g}, \tilde{J})$. Then lightlike vector field $\psi$ is parallel on $N$ iff

i) $\hat{N}$ is totally geodesic on $\tilde{N}$,

ii) $\tau=0$.

Proof. Let $\psi$ be a parallel vector field. In view of (3.1) and (3.33), for $U \in \Gamma(T N)$, we get

$$
\begin{aligned}
0 & =-\varphi A_{E}^{*} U-\tau(U) \psi \\
& =-\tilde{J} A_{E}^{*} U-\tau(U) \psi+u\left(A_{E}^{*} U\right) N
\end{aligned}
$$

Applying $\tilde{J}$ to (3.35) and from (3.1) with (2.3), we find

$$
\begin{aligned}
& -p \varphi\left(A_{E}^{*} U\right)-p u\left(A_{E}^{*} U\right) N-q A_{E}^{*} U \\
& -p \tau(U) \psi-q \tau(U) E-u\left(A_{E}^{*} U\right) \zeta \\
= & 0 .
\end{aligned}
$$

Taking tangential and transversal part of equation (3.36), we obtain

$$
q A_{E}^{*} U=-q \tau(U) E-u\left(A_{E}^{*} U\right) \zeta, \quad p u\left(A_{E}^{*} U\right)=0,
$$

which yields

$$
A_{E}^{*} U=0, \quad \tau(U)=0
$$

Theorem 3.4. Let $N$ be a screen semi-invariant lightlike hypersurface of a locally metallic semi-Riemannian manifold $(\tilde{N}, \tilde{g}, \tilde{J})$. Then lightlike vector field $\zeta$ is parallel on $N$ iff $N$ and $S(T N)$ is totally geodesic on $\tilde{N}$.

Proof. Since $\zeta$ is a parallel vector field, by use of (3.1) and (3.32), for $U \in \Gamma(T N)$ we get

$$
\begin{aligned}
0 & =-\varphi A_{N} U-\tau(U) \zeta \\
& =-\tilde{J} A_{N} U-\tau(U) \zeta+u\left(A_{N} U\right) N .
\end{aligned}
$$

Applying $\tilde{J}$ to (3.37) and using (3.1) with (2.3), we have

$$
\begin{aligned}
& -p \varphi\left(A_{N} U\right)-p u\left(A_{N} U\right) N-q A_{N} U \\
& -p \tau(U) \zeta-q \tau(U) N-u\left(A_{N} U\right) \zeta \\
= & 0 .
\end{aligned}
$$

Therefore, from (3.38), we obtain

$$
q A_{N} U=u\left(A_{N} U\right) \zeta, \quad p u\left(A_{N} U\right)=q \tau(U) .
$$

So we get the proof of our assertion.

Definition 3.4. Let $N$ be a screen semi-invariant lightlike hypersurface of a locally metallic semi-Riemannian manifold $(\tilde{N}, \tilde{g}, \tilde{J})$. If $B(U, V)=0$, for any $U \in \Gamma(\stackrel{D}{)})$ and $V \in \Gamma\left(\stackrel{\circ}{D}^{\prime}\right)$, then $N$ is called a mixed geodesic lightlike hypersurface.

Theorem 3.5. Let $N$ be a screen semi-invariant lightlike hypersurface of a locally metallic semi-Riemannian manifold $(\tilde{N}, \tilde{g}, \tilde{J})$. Then $N$ is a mixed geodesic lightlike hypersurface if and only if

i) There is no component of $A_{N}, \stackrel{\circ}{D}$-valuable. 
ii) There is no component of $A_{E}^{*}, \stackrel{\circ}{D}^{\prime}$-valuable.

Proof. Suppose that $N$ is mixed geodesic, i.e.,

$$
B(U, \zeta)=0 .
$$

By use of (2.4) and (2.11) in (3.39), we get

$$
\begin{aligned}
0 & =B(U, \zeta)=B(U, \tilde{J} N) \\
& =\tilde{g}\left(\tilde{\nabla}_{U} \tilde{J} N, E\right) \\
& =\tilde{g}\left(\left(\tilde{\nabla}_{U} \tilde{J}\right) N+\tilde{J} \tilde{\nabla}_{U} N, E\right) \\
& =\tilde{g}\left(\tilde{\nabla}_{U} N, \tilde{J} E\right) \\
& =-\tilde{g}\left(A_{N} U, \tilde{J} E\right),
\end{aligned}
$$

from which we obtain $(i)$.

Since

$$
-\tilde{g}\left(A_{N} U, \tilde{J} E\right)=\tilde{g}\left(A_{E}^{*} U, \tilde{J} N\right)
$$

we arrive at $(i i)$.

Now, we consider the distribution $\mu_{0}$, defined in (3.21). In view of (3.22) and taking

$$
\beta=\left\{\tilde{J}\left(\operatorname{Rad}\left(T N^{\prime}\right)\right) \oplus \tilde{J}\left(\operatorname{ltr}\left(T N^{\prime}\right)\right)\right\} \perp \operatorname{Rad}\left(T N^{N}\right),
$$

for any $U \in \Gamma\left(T N^{\prime}\right), V \in \Gamma\left(\mu_{0}\right)$ and $Z \in \Gamma(\beta)$, we can state

$$
\begin{aligned}
& \nabla_{U} V={\stackrel{\mu_{0}}{\nabla}}_{U} V+\stackrel{\mu_{0}}{h}(U, V), \\
& \nabla_{U} Z=-{\stackrel{\mu}{A_{0}}}_{Z} U+\nabla_{U}^{\perp} Z,
\end{aligned}
$$

where $\stackrel{\mu_{0}}{\nabla}$ is a linear connection on $\mu_{0}, \stackrel{\mu_{0}}{h}: \Gamma\left(T N^{\prime}\right) \times \Gamma\left(\mu_{0}\right) \rightarrow \Gamma(\beta)$ is an $\Im\left(N^{\prime}\right)$ bilinear, $\stackrel{\mu_{0}}{A}$ is an $\Im(N)$ linear operator on $\Gamma\left(\mu_{0}\right)$, respectively, and $\nabla^{\perp}$ is a linear connection on $\beta$.

Let $\wp \subset N^{\prime}$ be a coordinate neighborhood. Then according to decomposition given by (3.22), we take

$$
\begin{aligned}
& \alpha_{1}(U, V)=g\left(\stackrel{\mu_{0}}{h}(U, V), \tilde{J} N\right), \\
& \alpha_{2}(U, V)=g\left(\stackrel{\mu_{0}}{h}(U, V), \tilde{J} E\right), \\
& \alpha_{3}(U, V)=g(h(U, V), N),
\end{aligned}
$$

for every $U, V \in \Gamma\left(\left.\mu_{0}\right|_{\wp}\right)$. Thus we can write equation (3.40) by

$$
\nabla_{U} V={\stackrel{\mu_{0}}{\nabla}}_{U} V+\frac{1}{q} \alpha_{1}(U, V) \tilde{J} E+\frac{1}{q} \alpha_{2}(U, V) \tilde{J} N+\alpha_{3}(U, V) E .
$$

We shall compute $\alpha_{1}, \alpha_{2}$ and $\alpha_{3}$ in terms of $B$ and $C$. Starting with

$$
\begin{aligned}
g\left(\nabla_{U} V, \tilde{J} N\right) & =g\left(\nabla_{U} V+\frac{1}{q} \alpha_{1}(U, V) \tilde{J} E+\frac{1}{q} \alpha_{2}(U, V) \tilde{J} N+\alpha_{3}(U, V) E, \tilde{J} N\right) \\
& =\alpha_{1}(U, V) .
\end{aligned}
$$


Then by use of (2.10) and (2.11), we get

$$
\begin{aligned}
g\left(\nabla_{U} V, \tilde{J} N\right) & =g\left(\tilde{J} \nabla_{U} V, N\right) \\
& =g\left(\tilde{J} \tilde{\nabla}_{U} V, N\right) \\
& =g\left(\left(\tilde{\nabla}_{U} \tilde{J}\right) V+\tilde{J} \tilde{\nabla}_{U} V, N\right) \\
& =g\left(\tilde{J} \tilde{\nabla}_{U} V, N\right) \\
& =-g\left(A_{N} U, \tilde{J} V\right)=-C(U, \tilde{J} V) .
\end{aligned}
$$

Next we find

$$
\begin{aligned}
g\left(\nabla_{U} V, \tilde{J} E\right) & =g\left(\stackrel{\mu}{0}_{U} V+\frac{1}{q} \alpha_{1}(U, V) \tilde{J} E+\frac{1}{q} \alpha_{2}(U, V) \tilde{J} N+\alpha_{3}(U, V) E, \tilde{J} E\right) \\
& =\alpha_{2}(U, V),
\end{aligned}
$$

and from (2.10) and (2.13), we obtain

$$
\begin{aligned}
g\left(\nabla_{U} V, \tilde{J} E\right) & =g\left(\tilde{J} \nabla_{U} V, E\right) \\
& =g\left(\tilde{J} \tilde{\nabla}_{U} V, E\right) \\
& =g\left(\left(\tilde{\nabla}_{U} \tilde{J}\right) V+\tilde{J} \tilde{\nabla}_{U} V, E\right) \\
& =g\left(\tilde{J} V, \tilde{\nabla}_{U} E\right)=B(U, \tilde{J} V) .
\end{aligned}
$$

By a similar way, we compute

$$
\begin{aligned}
g\left(\nabla_{U} V, N\right) & =g\left(\stackrel{\mu}{0}_{U} V+\frac{1}{q} \alpha_{1}(U, V) \tilde{J} E+\frac{1}{q} \alpha_{2}(U, V) \tilde{J} N+\alpha_{3}(U, V) E, N\right) \\
& =\alpha_{3}(U, V),
\end{aligned}
$$

and

$$
g\left(\nabla_{U} V, N\right)=-C(U, V) .
$$

So, we can rewrite equation (3.43) with

$$
\nabla_{U} V=\stackrel{\mu}{\nabla}_{U} V-C(U, \tilde{J} V) \tilde{J} E-B(U, \tilde{J} V) \tilde{J} N-C(U, V) E
$$

Theorem 3.6. Let $N$ be a screen semi-invariant lightlike hypersurface of a locally metallic semi-Riemannian manifold $(\tilde{N}, \tilde{g}, \tilde{J})$. Then $\mu_{0}$ is integrable if and only if (3.45) $\quad C(\tilde{J} U, V)=C(U, \tilde{J} V), \quad B(\tilde{J} U, V)=B(U, \tilde{J} V), \quad C(U, V)=C(V, U)$, for every $U, V \in \Gamma\left(\mu_{0}\right)$.

Proof. Because of $\nabla$ is linear connection, by using (3.44) we get

$$
\begin{aligned}
{[U, V]=} & \stackrel{\mu}{0}_{U} V-\stackrel{\mu}{0}_{V} U \\
& +(C(U, \tilde{J} V)-C(\tilde{J} U, V)) \tilde{J} E \\
& +(B(U, \tilde{J} V)-B(\tilde{J} U, V)) \tilde{J} N \\
& +(C(U, V)-C(V, U)) E .
\end{aligned}
$$

If $\mu_{0}$ is integrable then the components of $[U, V]$ with respect to $\tilde{J} E, \tilde{J} N$ and $E$ vanish. So, we get proof of our asssertion.

Conversely, if (3.45) is satisfied we get

$$
[U, V] \in \Gamma\left(\mu_{0}\right) .
$$


Theorem 3.7. Let $N$ be a screen semi-invariant lightlike hypersurface of a locally metallic semi-Riemannian manifold $(\tilde{N}, \tilde{g}, \tilde{J})$. Then the distribution $\stackrel{\circ}{D}$ is integrable if and only if

$$
B(\tilde{J} U, \tilde{J} V)=p B(V, \tilde{J} U)+q B(V, U),
$$

for every $U, V \in \Gamma(\stackrel{\circ}{)})$.

Proof. Taking $U, V \in \Gamma(\stackrel{\circ}{D})$, we get $\tilde{J} U \in \Gamma(\stackrel{D}{D})$. Then $\stackrel{\circ}{D}$ is integrable iff

$$
\begin{aligned}
\tilde{g}([\tilde{J} U, V], \tilde{J} E)= & \tilde{g}\left(\tilde{\nabla}_{\tilde{\tilde{J}} U} V, \tilde{J} E\right)-\tilde{g}\left(\tilde{\nabla}_{V} \tilde{J} U, \tilde{J} E\right) \\
= & \tilde{g}\left(\tilde{J} \tilde{\nabla}_{\tilde{J} U} V, E\right)-\tilde{g}\left(\tilde{J} \tilde{\nabla}_{V} U, \tilde{J} E\right) \\
= & \tilde{g}\left(-\left(\left(\tilde{\nabla}_{\tilde{J} U} \tilde{J}\right) V+\tilde{\nabla}_{\tilde{J} U} \tilde{J} V, E\right)\right. \\
& -p \tilde{g}\left(\tilde{\nabla}_{V} U, \tilde{J} E\right)-q \tilde{g}\left(\tilde{\nabla}_{V} U, E\right) \\
= & \tilde{g}\left(\tilde{\nabla}_{\tilde{\tilde{J}} U} \tilde{J} V, E\right)-p \tilde{g}\left(\tilde{\nabla}_{V} U, \tilde{J} E\right)-q \tilde{g}\left(\tilde{\nabla}_{V} U, E\right) \\
= & B(\tilde{J} U, \tilde{J} V)-p B(V, \tilde{J} U)-q B(V, U),
\end{aligned}
$$

which gives (3.46).

Theorem 3.8. Let $N$ be a screen semi-invariant lightlike hypersurface of a locally metallic semi-Riemannian manifold $(\tilde{N}, \tilde{g}, \tilde{J})$. Then $\stackrel{\circ}{D}$ is parallel iff $\stackrel{\circ}{D}$ is totally geodesic on $N$.

Proof. From the definition of the distribution $\stackrel{\circ}{D}$ we know that $\stackrel{\circ}{D}$ is parallel if and only if

$$
g\left(\nabla_{U} V, \psi\right)=0
$$

So, we have

$$
\begin{aligned}
0 & =g\left(\nabla_{U} V, \psi\right) \\
& =\tilde{g}\left(\nabla_{U} V, \psi\right) \\
& =\tilde{g}\left(\nabla_{U} V, \tilde{J} E\right) \\
& =\tilde{g}\left(\tilde{J} \nabla_{U} V, E\right) \\
& =\tilde{g}\left(-\left(\left(\tilde{\nabla}_{U} \tilde{J}\right) V+\tilde{\nabla}_{U} \tilde{J} V, E\right)\right. \\
& =\tilde{g}\left(\tilde{\nabla}_{U} \tilde{J} V, E\right)=B(U, \tilde{J} V) .
\end{aligned}
$$

Thus we prove the assertion.

Definition 3.5. Let $N$ be a lightlike hypersurface of a locally metallic semiRiemannian manifold $(\tilde{N}, \tilde{g}, \tilde{J})$. If for every $U, V \in \Gamma(T N)$

$$
C(U, \omega V)=\phi B(U, V),
$$

or $A_{N}=\phi A_{E}^{*}$, then $N$ is called screen conformal lightlike hypersurface, where $\phi$ is a non-vanishing smooth function [4].

From (3.34) with (3.47), we find

$$
B(U, \zeta+\phi \psi)=0 .
$$

Theorem 3.9. Let $N$ be a screen conformal screen semi-invariant ightlike hypersurface of a locally metallic semi-Riemannian manifold $(\tilde{N}, \tilde{g}, \tilde{J})$. If $\tilde{N}$ or screen distribution $S(T N)$ is totally umbilical then $N$ is totally geodesic in $\tilde{N}$. 
Proof. Let $N$ be totally umbilical. Then we know that

$$
B(U, V)=\lambda g(U, V) \text {. }
$$

From (3.48), we get

$$
\lambda g(U, \zeta+\phi \psi)=0
$$

Putting $U=\psi$ in (3.50) we have $\lambda=0$ and $U=\zeta$ in (3.50) we have $\lambda \phi=0$. So we get $B=0=C$.

Now, if $S(T N)$ is totally umbilical we arrive at $B=0=C$.

\section{REFERENCES}

[1] K. L. Duggal, A. Bejancu, Lightlike submanifolds of semi-Riemannian manifolds and applications, Mathematics and Its Applications. Kluwer Publisher. (1996).

[2] K. L. Duggal, B. Şahin, Generalized Cauchy-Riemann lightlike submanifolds of Kaehler manifolds. Acta Math. Hungarica. 112, 107-130 (2006).

[3] K. L. Duggal, B. Şahin, Lightlike submanifolds of indefinite Sasakian manifolds. Int. J. Math. and Math. Sci. Article ID 57585 (2007).

[4] K. L. Duggal, B. Şahin, Differential geometry of lightlike submanifolds. Frontiers in Mathematics. (2010).

[5] G. Galloway, Lecture notes on spacetime geometry. Beijing Int. Math. Research Center. 1-55 (2007).

[6] C. E. Hretcanu, M. C. Crasmareanu, Applications of the golden ratio on Riemannian manifolds. Turkish J. Math. 33 (2), 179-191 (2009).

[7] F. E. Erdoğan,C. Yıldırım, Semi-invariant submanifolds of golden Riemannian manifolds. AIP Conference Proceedings. 1833, 020044 doi:10.1063/1.4981692 (2017).

[8] B. E. Acet, S. Yüksel Perktaş, E. Kılıç, Symmetric lightlike hypersurfaces of a para-Sasakian space form. Analele Stiintifice Ale Universitatii Al.I. Cuza Iasi (N.S.) 2, 915-926 (2016).

[9] B. E. Acet, S. Yüksel Perktaş, E. Kılıç, On lightlike geometry of para-Sasakian manifolds. Scientific Work J. Article ID 696231, (2014).

[10] F. E. Erdoğan and C. Yıldırım, Lightlike Submanifolds with Planar Normal Section in Semi-Riemannian Product Manifolds, Intenational Electronic Journal of Geometry, Vol9, No1,(2016),70-77.

[11] C. Yıldırım and B. Şahin, Screen Transversal Lightlike submanifolds of indefinite Sasakian Manifolds, An.Şt. Univ. Ovidius Constanta, Vol.18(2), (2010), 315-336.

[12] F. E. Erdoğan, R. Gunes and B. Şahin, Half-lightlike submanifold with planar normal sections in R42, Turk. J. Math. Vol.38, (2014), 764-777.

[13] N. Ö. Poyraz, and E. Yaşar, Lightlike Hypersurfaces of A Golden semi-Riemannian Manifold, Mediterr. J. Math.(2017)14:204.

[14] S. Y. Perktaş, E. Kılıç, and B. E. Acet, Lightlike Hypersurfaces of Para-Sasakian Space Form, Gulf J. Math. 2(2), (2014),333-342.

[15] V. W. de Spinadel, On characterization of the onset to chaos, Chaos, Solitons \& Fractals 8(10) (1997) 1631-1643.

[16] V. W. de Spinadel, The metallic means family and forbidden symmetries, Int. Math. J. 2(3) (2002) 279-288.

[17] V. W. de Spinadel, The metallic means family and renormalization group techniques, Proc. Steklov Inst. Math., Control in Dynamic Systems, suppl. 1 (2000) 194-209.

[18] F. E. Erdoğan and C. Yıldırım, On a Study of the Totally Umbilical SemiInvariant Submanifolds of Golden Riemannian Manifolds, Journal of Polytechnic, DOI10.2339/politeknik.389629.

[19] F. E. Erdoğan, B. Şahin and R. Gunes, Lightlike surfaces with planar normal sections in Minkowski 3-Spaces, Int. Electron. J. Geom. 7(1) 133-142, 2014.

[20] A. P. Stakhov, B. Rozin, The "golden" algebraic equations, Chaos, Solitons \& Fractals 27(5) (2006) 1415-1421.

[21] S. I. Goldberg and K. Yano, Polynomial structures on manifolds, Kodai Math. Sem. Rep. 22 (1970) 199-218. MR0267478. 
[22] S. I. Goldberg and N.C. Petridis, Differentiable solutions of algebraic equations on manifolds, Kodai Math. Sem. Rep. 25 (1973), 111-128. MR0315627.

[23] C. E. Hretcanu and M. Crasmareanu, On some invariant submanifolds in a Riemannian manifold with Golden structure, An. Stiint. Univ. Al. I. Cuza Iasi. Mat. (N.S.) 53 (2007), suppl. 1, 199-211. MR2522394.

[24] M. Crasmareanu and C. E. Hretcanu, Golden differential geometry, Chaos Solitons Fractals 38 (2008), no. 5, 1229-1238. MR2456523.

[25] M. Crasmareanu, C. E. Hretcanu and M. I. Munteanu, Golden- and product-shaped hypersurfaces in real space forms, Int. J. Geom. Methods Mod. Phys. 10 (2013), no. 4, 1320006, 9 pp. MR3037234.

[26] C. E. Hretcanu and M. Crasmareanu, Metallic structures on Riemannian manifolds, Rev. Un. Mat. Argentina 54 (2013), no. 2, 15-27. MR3263648.

[27] A. Gezer, N. Cengiz, On integrability of Golden Riemannian structures, Turkish J. Math. 37(2013), 693-703.

[28] C. E Hretcanu, A. M. Blaga, Submanifolds in metallic Riemannian manifolds, arXiv preprint arXiv:1803.02184, 2018 - arxiv.org.

[29] C. E. Hretcanu, A. M. Blaga, Slant and semi-slant submanifolds in metallic Riemannian manifolds, arXiv preprint arXiv:1803.03034, 2018 - arxiv.org.

[30] A. M. Blaga, C. E. Hretcanu, Invariant, anti-invariant and slant submanifolds of a metallic Riemannian manifold, arXiv preprint arXiv:1803.01415, 2018 - arxiv.org.

Faculty of Arts and Sciences, Department of Mathematics, Adiyaman University, AdiYAMAN, TURKEY

E-mail address: eacet@adiyaman.edu.tr 